eintreten, oder wo sich der septicämische Cliarakter der Infection, d. h. das Uebergreifen derselben auf das Blut geltend macht. Beim Auftreten der ersten localen Erscheinungen der Infection, der Schwellung und Röthung, des Juckens an der Infectionsstelle, lässt sich niemals voraussagen, welchen Charakter die Infection annehmen wird, ob sie local bleiben, oder sich auf die weitere Umgebung oder auf die benachbarten Lymphdrüsen oder gar auf Blut und innere Organe verbreiten wird.

Bei dieser Ungewissheit über die weitere Entwickelung der Infection erscheinen auf den ersten Blick alle diejenigen Maassnahmen geboten, welche eine radicale Entfernung der erkrankten Stelle durch Exstirpation, durch Ausbrennen oder eventuell durch Absetzung der der Impfstelle zugehörigen Extremität erstreben. Zur Motivirung einer derartigen Therapie muss aber vorher die Frage entschieden sein: Isteswirklich möglich, zu der Zeit, wo die Diagnose "Milzbrand" sicher feststebt, durch Entfernung der Impfstelle sämmtliche Milzbrandkeime aus dem Körper zu entfernen, oder sind schon durch Blut- oder Lymphbahn einzelne Keime von der Infectionsstelle nach dem Körperinnern geschafft, wo sie unbekümmert um die am Locus infectionis sich abspielenden Vorgänge weiter wachsen können?

Genügt doch bekanntlich, wie experimentell dargethan ist, eine ganz geringe Zahl von Milzbrandbacterien, vielleicht oder besser wahrscheinlich ein einziger virulenter Bacillus oder eine einzige virulente Spore, um wenigstens beim Kaninchen oder Meerschweinchen den Tod an Milzbrand herbeizuführen. Will man nun die oben anfgestellte Frage, ob ein sofortiger chirurgischer Eingriff in jedem Falle ausreichend ist, oder ob er nur anf die zur Generalisation neigenden Fälle zu beschränken oder bezüglich dieser zu fordern sei, einer experimentellen Beantwortung unterziehen, so wird gerade das eben erwähnte Verhalten obiger Versuchsthiere als besonders geeignet anzusehen sein. Denn da man bei diesen Versuchen den Zeitpunkt der Impfung und den Zeitpunkt der radicalen Entfernung der Impfstelle genau fixiren kann, so werden, wenn innerhalb dieser beiden Zeitpunkte auch nur eine ganz geringe Anzahl von Milzbrandkeimen jenseits der entfernten Partie gelangt sind, diese immer die Allgemeininfection, den Tod der Versuchsthiere herbeiführen.

Die diesbezügliche Versuchsreihe wurde nach folgendem Plane angestellt:

Impfung mehrerer Versuchsthiere an der äussersten Stelle einer Extremität mit virulentem Milzbrandmaterial - Absetzung der gesammten Extremität möglichst weit entfernt von der Infectionsstelle, bei den verschiedenen Thieren nach verschiedener Zeit - nachfolgende Beobachtung derThiere. Bei eintretendem Tod bacteriologische Untersuchung des Blutes und der inneren Organe auf Milzbrandbacillen.

Zu den Versuchen wurden grosse kräftige Kaninchen gewählt

IV. Aus der Königl. chirurgischen Universitätsklinik zu Halle a. S.

\section{Ein experimenteller Beitrag zur Frage der Milzbrandbehandlung.}

Von Dr. Franz Nissen, Assistenten der Klinik.

Wåhrend in der Behandlung der durch die pyogenen Bacterien herbeigeführten Entzündungen und Eiterungen im allgemeinen eine vollständige Uebereinstimmung herrscht, insofern als nach dem Grundsatz ubi pus, ibi evacua dem Messer die Freilegung der erkrankten Partieen überlassen wird, sind die Meinungen über die Behandlung der Milzbrandkrankheit getheilt; von der einen Seite wird eine abwartende symptomatische Therapie, von der anderen Injectionen von Antisepticis in die erkrankten Partieen, von einer dritten schliesslich Incision oder Excision und Kauterisation des erkrankten Gebietes anempfohleı. Wir sehen selbstverständlich hier von dem sogenannten Darmmilzbrand $a b$, d. h. derjenigen Form derErkrankung, welche den primären Sitz auf der Intestinalschleimhaut hat, sondern beschäftigen uns im folgenden nur mit dem in der äusseren Haut und den zugänglichen Schleimhautpartieen localisirten Milzbrand, dem Milzbrandcarbunkel, der Pustula maligna.

Die Milzbrandinfection tritt beim Menschen in vielen Fällen nicht in der bösartigen Form anf, wie sie nach Infection des Versuchsthieres mit virulentem Material gewöhnlich ist, mit einer Vermehrung der Milzbrandbacterien in dem gesammten Gefässsystem, einer Ablagerung derselben in den parenchymatösen Organen und einer schliesslich den Tod bedingenden Verstopfung der kleinsten Gefässe; vielmehr beschränkt er sich oft nur auf die Bildung kleiner unschuldiger Pusteln, welche in ihrem Verlaufe einfachen Furunkeln āhnlich sein können. Andererseits giebt es auch Fälle, in denen an der Eingangspforte des Milzbrandvirus bedrohliche mit hohem Fieber und schwerem Collaps einhergehende locale Erscheinungen

) Arch. f. Gyn. Bd. XXXI. H. 2. als Infectionsstelle diente anfangs die äusserste Spitze des Ohres, welches nach verschiedenen Zeitintervallen $(1 / 2,3 / 4,1,2,5$ u. s. w. Stunden) an der Basis mittels des Paquelins abgebrannt wurde.

Später wurdeis als Impfstellen auch die Enden der oberen und unteren Extremitäten genommen und in verschiedenen Zeiträumen die ganzen Extremitäten möglichst nach der Achselhöhle beziehungsweise Leistenbenge blutig abgesetzt. Der zur Impfung benutzte Milzbrand zeigte volle Virulenz, tödtete Mäuse in 18 bis 24 Stunden, Meerschweinchen in 24 bis 36 , Kaninchen in 24 bis 48 Stunden. Entweder wurde mit sporenhaltiger bei $38^{\circ} \mathrm{C}$ gezüchteter Agarcultur, oder mit dem Blut eben an diesem Milzbrand verendeter Mäuse geimpft.

$$
\text { Einige Beispiele. }
$$

I. Kaninchen A, B, C, D werden an der Spitze eines Ohres unter sorgfältigster Vermeidung jeder Blutung mit je einer Platinöse frischen Milzbrandblutes geimpft.

Bei A wird nach 4 Stunden, bei B wird nach 14 Stunden, bei C wird nach 20 Stunden das geimpfte Ohr an der Wurzel mittels des Themokauters abgesetzt.

D dient zur Controlle.

Alle 4 Kaninchen werden im Verlaufe von 24-36 Stunden nach der Impfung todt gefunden. Als Todesursache wird durch mikroskopische Ontersuchung und Verimpfung des Blutes auf Mäuse „Milzbrand" festgestellt.

II. Kaninchen A, B, C wie in Vers. I geimpft.

Bei $A$ und $B$ wird nach einer Stunde das geimpfte Ohr an der Wurzel abgesetzt. endet.

A und B bleiben am Leben. C nach 28 Stunden an Milzbrand ver-

III. Kaninchen A, B, $\mathrm{C}$ an der Ohrspitze, D an der Fussspitze mit frischem Milzbrandblut geimpft.

Bei $\mathrm{A}$ und $\mathrm{B}$ nach $3 / 4$ Stunden das 0 hr abgesetzt, bei $\mathrm{D}$ wird nach $3 / 4$ Stunden der Oberschenkel abgesetzt.

$\mathrm{C}$ dient zur Controlle. A wird ebenso wie das Controllthier $\mathrm{C}$ nach 48 Stunden todt gefunden. Ursache: Milzbrand. B und D bleiben am Leben.

IV. Kaninchen A an der Ohrspitze, B an der Fussspitze, $\mathrm{C}$ an der Fussspitze mit je einer Platinöse Milzbrandblut geimpft.

Bei A und B nach drei Stunden Ohr bezw. Oberschenkel abgesetzt. 
C dient zur Controlle. Nach 24 bis 48 Stunden alle drei Kaninchen an Milzbrand verendet. fassen :

Das Resnltat obiger Versnchsreihe können wir dahin znsammen-

Schon 2-3 Stunden nach der Impfnng lässt sich, trotz $\mathrm{Ab}$ setzung der der Impfstelle zngehörigen Extremität (Arm, Bein, Ohr) in möglichst weiter Entfernnng von derselben, der Tod des Versnchsthieres an Milzbrand nicht abwenden. Innerhalb dieser Zeit müssen also bereits Milzbrandkeime jenseits der Absetznngstelle gelangt sein.

Der Fall, dass ein Kaninchen, bei welchem $3 / 4$ Stnnden nach der Impfnng die Extremität (Ohr) abgesetzt wird, an Milzbrand zn Grnnde geht (Vers. III, Kaninchen B) steht nnter allen Beispielen einzig da.

Die Regelmåssigkeit einerseits nnd die relative Langsamkeit der Bacterienbewegnng andererseits, wie sie ans nnseren Versnchen hervorgeht, mnssten von vornherein einen Hinweis anf die Bahn geben, anf der die Bacillen oder Sporen fortgeschafft wnrden. Bei der Impfnng werden dnrch die Eröffnnng zahlreicher Blntcapillaren nnd Lymphspalten zwei Eingangspforten für die Bacterien geschaffen, die eine in die Blnt-, die andere in die Lymphcircnlation führend.

Injicirt man einige hnnderttansende von Milzbrandbacillen einem Kaninchen intravenös, so gelingt bereits nach 5-10 Minnten der Nachweis von Milzbrandkeimen im Blnt mittels des Cnltnrverfahreus nicht mehr. Die Milzbrandbacillen sind aber nicht etwa im circnlirenden Blute abgetödtet, sondern sie sind innerhalb dieser knrzen Zeit hauptsächlich in Leber, Milz nnd Knochenmark abgelagert worden; ihre Gegenwart und Lebensfähigkeit lässt sich leicht dnrch Züchtnng nachweisen. Würden demgemäss die Milzbrandbacillen bei der Impfnng in den BIntkreislanf eintreten, so müsste schon nach wenigen Minnten die Entfernnng der Impfstelle ansserstande sein, die Allgemeininfection $\mathrm{zn}$ verhäten. Dies ist.nnn in der That nicht der Fall, da, wie nusere Versnche lehren, zwei bis drei Stnnden nöthig sind, nm trotz Entfernnng der Infectionsstelle die Allgemeininfection herbeiznführen. Ansserdem erscheint eine Anfnahme der Milzbrandkeime in die Blntgefässe anch deshalb nnwahrscheinlich, weil in den Blntcapillaren nnd selbst in den be der Impfnng eventnell verletzten kleinen Venen der peripheren Korperstellen ein positiver Druck herrscht, welcher ein Heranspressen des Blntes nnd damit eine Entfernnng, nicht aber eine Ansangnng der in die Wnnde gebrachten Keime bewirkt; in den Lymphgefässen hingegen herrscht $e^{i n}$ negativer Drnck, dessen Folge eine Ansangnng der in der Wnude befindlichen Flüssigkeit nnd damit eine Anfnahme der Milzbrandkeime in die Lymphcircnlation ist.

Gelangen die Milzbrandkeime nnn wirklich in den Lymphstrom so müssen sie nothwendiger Weise die Lymphdrüsen passiren, nnd da hier eine ganz bedentende Verzögernng der Lymphbewegnng stattfindet, so musste es voranssichtlich gelingen, zn einer bestimmten Zeit nach der an den Extremitäten gesetzten Impfnng etwa vorhandene Milzbrandkeime in den Lymphdrüsen nachznweisen. Die Gegenwart schon einer ganz geringen Zahl von virnlenten Milzbrandkeimen mnsste sich also dnrch Verimpfnng der Lymphdrüsen anf absolnt empfängliche Thiere, z. B. weisse Mänse mit Sicherheit nachweisen lassen. ${ }^{1}$ )

Als Versnchsthiere dienten Kaninchen, späterhin anch Meerschweinchen. Als Impfmaterial sporenhaltige bei $380 \mathrm{C} \mathrm{3-4}$ Tage lang gezüchtete, immer von Milzbrandblnt abgeimpfte schräge Agarcultnren. Als Impfstellen wnrden die peripheren Theile der nnteren nnd oberen Extremitäten nnd der Ohren gewählt. Die Impfnng geschah entweder mittels Verreibnng von Cnltnr in einer snbcntanen Hanttasche oder selten dnrch snbcntane mit geringem Drnck bewirkte Injectionen von Anfschwemmnng einer Agarcnltnr in sterilisirtem Wasser.

Die den Infectionsstellen an den nnteren Extremitäten zunächst gelegenen Lymphdrüsen liegen beim Kaninchen nicht wie beim Menschen in der Leistenbenge, sondern weiter central in Rosenkranzform längs der Arteria nnd Vena iliaca, sich nnmittelbar in die retroperitonealen Drüsen fortsetzend: an der oberen Extremität liegen sie in Axillarfett, nnd an den Ohren anf der Ohrspeicheldrüse. Bei einiger Uebnng lassen sich die leicht rosa gefärbten, opak erscheinenden Drüschen leicht heranspräpariren, aber nnr nnter Vermeidnng jeglicher Blntnng, sonst sind die Drüschen schwer von den Fetttränbchen zn nnterscheiden.

Um bei den ersten Versnchen das Anffinden der Lymphdrüsen zn erleichtern, haben wir central von der Infectionsstelle fein verriebene chinesische Tnsche snbcntan injicirt; dnrch die Aufnahme der Farbstoffkörnchen bekommen die Drüschen ein granes Anssehen nnd sind dann leicht von dem nmgebenden Gewebe zn nnterscheiden. Bei den späteren Versnchen war dieses Hülfsmittel nicht mehr nöthig.

I) Von einer mikroskopischen Untersuchung der Lymphdrüsen war bei der starken Verdünnung, in welcher die Keime in denselben vorhanden sein mussten, a priori nichts zu erwarten.
Einige Beispiele.

V. Einem Kaninchen wird je 0,5 ccm einer Aufschwemmung von sporenhaltiger Milzbrandagarcultur in sterilisirter Emulsion chinesischer Tusche in der Vorderseite des Unterschenkels subcutan mit geringem Druck injicirt.

Nach $1 \frac{1}{2}$ Stunden werden die auf den Iliacalgefässen liegenden Lymphdrüsen frei gelegt und zwei weissen Mäusen unter die Hant gebracht.

Die Măuse sind am dritten Tage an Milzbrand verendet.

VI. Einem Kaninchen wird je $0,5 \mathrm{ccm}$ einer Aufschwemmung sporenhaltiger Agarcultur in sterilisirtem Wasser in die Vorderseite beider Unterschenkel subcutan mit geringem Druck injicirt.

Nach 2 Stunden werden die auf den grossen Schenkelgefässen liegenden Lymphdrüsen drei Mäusen unter die Haut gebracht.

Alle drei Mäuse sind nach drei Tagen an Milzbrand verendet.

VII. Einem Kaninchen wird in einer am Unterschenkel (beiderseits) angelegten Hauttasche je eine volle Platinöse sporenhaltiger Agarcultur verrieben. Am Oberschenkel wird gleichzeitig subcutan 0,5 einer sterilisirten Aufschwemmung chinesischer Tusche injicirt.

Die nach $1 \frac{1}{2}$ Stunden exstirpirten Lymphdrüsen werden vier weissen Mäusen unter die Rückenhaut gebracht.

Die Măuse sind nach 14 Tagen noch am Leben.

VIII. Einem Kaninchen wird in einer an beiden Unterschenkeln und beiden Unterarmen angelegten Hauttasche je eine volle Platinöse sporenhaltiger Agarcultur verrieben. Nach 4 Stunden werden auf den grossen Schenkelgefässen und die in der Achselhöhle liegenden Lymphdrüsen auf sechs weisse Mäuse verimpft.

Nach zwei Tagen sind alle sechs Mäuse todt. Ursache: Milzbrand.

Wir haben also folgende Beobachtnng: Bei snbcntaner Injection von $0,5 \mathrm{ccm}$ einer leicht getrübten Sporenanfschwemmnng in den periphersten Theil der nnteren Extremität konnte man schon nach $\mathbf{1}^{1 / 2}$ Stnnden Milzbrandkeime in den anf den grossen Gefässen des Beckens liegenden Lymphdrüsen dnrch Verimpfnng derselben anf weisse Mänse nachweisen. Wnrde die Agarcnltnr snbcntan verrieben, so fand man 3-4 Stunden nach der Impfnng Milzbrandkeime in den vächstgelegenen Lymphdrüsen. Die Mänse starben, wenn die verimpften Drüsen infectionsfăhiges Material enthielten, gewöhnlich 2-3 Tage nach der Impfnng, während die nrsprüngliche Reincnltnr meist innerhalb 24 Stnnden den Tod der geimpften Mänse herbeiführte. Die schnellere Anknnft der Bacterien in den Lymphdrüsen bei der snbcntanen Injection ist wohl nnr aus einem rein mechanischen Moment, ans der Vorwärtstreibnng der Keime dnrch den Druck der Spritze nnd der elastischen Beschaffenbeit der gespannten Hant zn erklären.

An der oberen Extremität nnd am Ohr differiren die entsprechenden Zeiten wenig von denen an der nnteren Extremität.

Darans folgt: Drei bis vier Stnnden nach der an der nnteren Extremität stattgehabten Impfnng sind Milzbrandkeime bereits in den nächstgelegenen Lymhdrüsen angelangt.

Ziehen wir nnn das Resnmé nnserer Versnche bei Kaninchen nnd Meerschweinchen, so ergiebt sich, dass bereits wenige Stnnden nach der Impfnng an den peripheren. Theilen der Extremitäten die Milzbrandkeime in den nächstgelegenen Lymphdrüsen angelangt sind, nnd dass ans diesem Grnnde eine, wenige Stnnden nach der Impfnng vorgenommene hohe Abtragnng der Extremität nicht imstande ist, den Tod des Versnchsthieres abznwenden.

Ferner folgt ans nuseren Versnchen, dass die Infectionsstelle selbst für die Weiterverbreitnng der Infection, etwa dadnrch, dass daselbst immer nene Milzbrandkeime gebildet nnd in die Blntbahn nnd inneren Organe fortgeschafft werden, ohne Bedentnng ist, da schon $z$ einer Zeit, wo die Verändernngen am locns infectionis kanm sichtbar sind, wenige von der Infectionsstelle abgetrennte nnd in die Lymphcircnlation übergegangene Keime genügen, nm den Tod des Thieres an Milzbrand herbeiznführen.

Bevor wir diese Versnche anf die Verhältnisse beim Menschen n̈bertragen, wollen wir die einzelnen Momente der Milzbrandinfection bei Versnchsthier nnd Mensch vergleichen.

Znnächst haben wir keinen Grnnd, anznnehmen, dass beim Menschen die in eine Wnnde gelangten Milzbrandkeime in allgemeinen einen anderen Weg in das Innere des Körpers wählen, als beim Kaninchen, d. h. die Lymphbahn; hierfür spricht schon die mitnnter anftretende Entzündnng der nächstgelegenen Lymphdrüsen.

Bei der Uebereinstimmnng dieser Verhältnisse bei Versnchsthier nnd Mensch müssen wir nns die schon anfangs angedentete Frage stellen: "Ist denn wirklich zn dem Zeitpunkt, an welchem bei dem menschlichen Patienten dnrch das Mikroskop oder gar die Thierimpfung die Diagnose "Milzbrand" sicher gestellt oder dnrch Anamnese nnd klinische Erscheinnngen wahrscheinlich gemacht ist, dnrch radicale Entfernnng der Impfstelle eine vollständige Entfernnng aller Milzbrandkeime nnd eine Vermeidnng der Allgemeininfection zn erwarten?" Ganz entschieden müssen wir nach nnseren Versnchen diese Frage mit "nein" beantworten nnd anch beim Menschen annehmen, dass nach radicaler Entfernnng der Impfstelle Keime wenigstens in den benachbarten Lymphdrüsen znräckbleiben. Eine weitere Frage 
ist: Welche Bedeutung hat beim Menschen die Infectionsstelle für die Weiterentwickelung der Infection?

Beim Kaninchen genügen wenige in die Lymphbahn aufgenommene Bacillen, um die Infection zu unterhalten; die Infectionsstelle selbst hat auf die weitere Ausbildung der Krankheit keinen Einfluss; ihre Entfernung ist ohne therapeutischen Erfolg. Wie liegen nun diese Verhältnisse beim Menschen? Genügen auch hier wenige von der Infectionsstelle abgetrennte Keime, um die Infection weiter auszubilden, oder bedarf es hierzu einer massenhaften Neubildung von Keimen an der Impfstelle und deren Fortschaffung durch das Lymphgefässsystem in das Blut und die inneren Organe? In letzterem Falle wäre die Infectionsstelle von hoher Bedeutung für die Weiterentwickelung der Erkrankung, und ihre frühzeitige Entfernung müsste einen grossen therapeutischen Erfolg haben. Gegen eine derartige Anschauung spricht die klinische Erfahrung, unter anderem auch folgender gut beobachteter Fall von Milzbrandinfection im Laberatorium. ${ }^{1}$ ) Am zweiten Tage nach der vermeintlich bei einem Thierversuch stattgehabten Infection am Finger traten bereits die ersten localen Erscheinungen an der Impfstelle auf; am dritten Tage radicale Entfernung derselben mit dem Messer (durch Prof. Angerer), Auswaschung der Wunde und Umschläge mit Sublimatlösung 1:2000.

$\mathrm{Am}$ vierten Tage, ohne dass an den vorangegangenen Tagen eive Veränderung an den Lymphbahnen des Unter- und Oberarms und an den Lymphdrüsen der Ellenbogengegend bemerkt worden wäre, Anschwellung der axillaren Lymphdrüsen. Am fünften Tage Aussehen der Wunde reactionslos, Entfernung der afficirten Lymphdrüsen (durch Prof. Nussbaum); in Blut und Lymphdrüsen Milzbrandkeime durch Züchtung nachweisbar. Heilung. - Trotz der radicalen Entfernung der Impfstelle - wie aus dem reactionslosen Verhalten derselben hervorgeht - hat die bereits in die Lymphdrüsen fortgeschaffte Bacillenzahl genügt, um die Infection weiter zu erhalten.

Hier hatte also die Entfernung der Infectionsstelle keinerlei Einfluss auf die Verbreitung der Infection auf die Lymphdrüsen.

Im Gegensatz hierzu hatten wir Gelegenheit, einen Fall von Milzbrandinfection zu beobachten, der zwar sehr heftige locale Erscheinungen machte, bei welchem aber trotz abwartender Behandlung weder eine makroskopisch wahrnehmbare Betheiligung der benachbarten Lymphdrüsen, noch eine Erkrankung des Blutes bei öfterer Untersuchung constatirt werden konnte. Der inficirende Milzbrand wurde rein gezüchtet und zeigte sich für Kaninchen, Meersch weinchen und Mäuse in hohem Grade virulent. Es handelte sich hier um einen Schäfer, der am 28. August 1890 ein Stück Fleisch von einem gefallenen Schaf als "Hundefutter" erhalten, mit seinen Händen angefasst, in Papier gewickelt und nach Hause gebracht, dort gekocht und seinem Hund als Futter gegeben hatte. Am 29. empfand Patient Jucken an der rechten-Wange und kratzte die Stelle offer mit dem Fingernagel. Am Abend Schwellung und R.othung der Kratzstelle. Allgemeinbefinden noch gut. Am 30. starke Anschwellung der Wange, der oberen und unteren Augenlider - Fiebererscheinungen. Am 31. Aufnahme in die Klinik. Status: Ueber dem rechten Jochbein eine etwa $1 \frac{1}{2} \mathrm{qcm}$ grosse, mit schwarzem fest aufsitzendem Belag bedeckte geschwürige Stelle, Oedem der rechten Nasenhälfte, der oberen und unteren Augeulider. Temperatur abends $40^{\circ} \mathrm{C}$; keine lymphangoitische Stränge, keine Anschwellung der Lymphdrüsen. Impfung je einer Platinöse Oedemflüssigkeit auf zwei Măuse hat den Tod derselben innerhalb 24 Stunden zur Folge. Todesursache Milzbrand. Züchtung der Oedemflüssigkeit auf Agaragarplatten ergiebt Colonieen von Staphylococcus albus und Milzbrand. Täglich ausgeführte Untersuchung des Blutes (zuerst mehrmals am Tage) mittels des Culturverfahrens hat negativen Erfolg. ${ }^{1}$ ) Ausgang in Heilung unter allmählicher Entfieberung und nach Abstossung der einen grösseren nekrotischen Hautpartie.

Während wir beim Meerschweinchen und bei Impfung mit einer ganz geringen Zahl von Milzbranderregern immer die in typischer Weise verlaufende Allgemeininfection hervorrufen können, tritt diese beim Menschen in seltenerem Falle ein. Eine Erklärung dafür ist wohl - vorausgesetzt, dass das inficirende Material volle Virulenz besitzt - in der Verschiedenheit der individuellen Disposition zu suchen; der eine reagirt nach der Infection mit localer, der andere mit localer Erkrankung unter Betheiligung der nächstgelegenen Lymphdrüse, bei dem dritten schliesslich werden noch Blut und innere Organe? in Mitleidenschaft gezogen.

1) Centralblatt für Bacteriol. $1889 \mathrm{Bd}: \mathrm{V}$ No. 21.

2) Tägliche Impfung mit der Oedemflüssigkeit verschiedener Stellen immer auf zwei bis drei Măuse ergiebt am 1. und 2. September positive, am 3. September negatives Resultat. Also bereits fünf Tage nach der wahrscheinlich stattgehabten Infection sind in der Omgebung der Impfstelle bei ${ }^{* *}$ noch vorhandenen Entzündungserscheinungen Milzbrandbacillen durch Thierimpfung nicht mehr nachzuweisen! Hier also Infection mit virulentem Milzbrand, Beschränkung derselben auf die Iufectionsstelle, keine Betheiligung der Lymphdrüsen und des 'Blutes;' dabei eine abwartende Behandlung Verband mit Unguentum cinereum.
Wenn wir uns zum Schluss die Frage vorlegen, wie wir uns beim Menschen gegenüber einer Milzbrandinfection der Haut bezüglich einer radicalen operativen Therapie verhalten sollen, so werden wir dieselbe verwerfen und eine abwartende Behandlung anempfehlen. Bei einer geringen localen Ausdehnung des Milzbrandes begrenzt sich die erkrankte Stelle bald von selbst - offenbar durch schnelles Absterben der Bacillen - bei grosser Ausdehnung der localen Erscheinungen ist eine Begrenzung operativ nicht möglich und schliesslich - dies ist der wichtigste Punkt - hat in keinem $d \in r$ beiden Fälle die operative Behandlung der Infectionsstelle irgend welchen Einfluss auf die Generalisation der Infection.

Die circuläre Abschnürung der Extremität als hemmendes Moment für die Bewegung der Bacterien.

Wie wir aus der voranstehenden Untersuchung schliessen kőnnen, muss es bei der Belrandlung ganz frisch mit Milzbrand inficirter Wunden als Hauptaufgabe zu betrachten sein, bis zu dem Termin, an welchem der Arzt mit seiner desinfectorischen Thätigkeit an die inficirte Wunde herantritt, jede Fortbewegung der aufgenommenen Milzbrandkeime von der Infectionsstelle weg zu verhindern, da ja - wenigstens beim Versuchsthier - schon ganz wenige von der Impfstelle abgetrennte Bacillen genügen, um unbekümmert um die sich an der Wunde abspielenden Vorgänge, die Milzbrandinfection weiter zu unterhalten. Gelingt es uns aber, alle aufgenommenen Milzbrandkeime an der Wunde zurückzuhalten, so werden wir dieselben mit Hülfe der uns zur Verfügung stehenden Desinfectionsmaassregeln leicht vernichten können.

Es ist ein altes probates Volksmittel, nach Schlangenbissen die verletzte Extremität oberhalb der Bisswunde sofort zu umschnüren und bis zum Eintritt der ärztlichen Behandlung umschnürt zu halten. Offenbar wird durch die feste Umschnürung des ganzen Gliedes ein sofortiger Stillstand der Blut- und Lymphcirculation hergestellt, welcher eine Fortschaffung des Giftes von der Wunde in die inneren Organe verhindert.

In ähnlicher Weise versuchte ich auf den Rath des Herrn Prof. v. Bramann, die Wirkung der circulären Abschnürung der Extremität auf das Verhalten der in eine peripher gelegene Wunde eingebrachten Milzbrandbacillen folgendermaassen festzustellen:

An dem Extremitätenende eines Kaninchens wurde eine oberflächliche Hautwunde gesetzt, die Wundfläche mit einer Platinöse frischen Milzbrandmäuseblutes bestrichen - damit die Milzbrandbacillen geschützt lagen, wurde die Haut in Form eines kleinen Läppchens abgelöst und auf die inficirte Stelle zurückgelegt - und sofort an dem höher gelegenen Theil der Extremität mittels eines Gunmischlauches fest umschnürt. Nach 3-4 Stunden wurde die ganze Infectionsstelle gründlich mit dem Paquelin ausgebrannt und der Gummischlauch entfernt. Sofort nach der Umschnürung schleppte das Thier das ganze Bein nach; die Parese der Muskulatur dauert noch 1 bis 2 Tage an, um dann ganz zu verschwinden. Bei allen a uf diese Weise behandelten Thieren blieb ausnahmslos die Milzbrandinfetion aus. Wurde hingegen der Schlauch gelöst, ohne dass irgend welche Desinfectionsmaassregeln an der Infectionsstelle getroffen waren, so gingen die Thiere ausnahmslos an Milzbrand nach 48 bis 60 Stunden zu Grunde, und zwar waren es alle die Thiere, welche bei der ersteren Art der Behandlung von jeder Erkrankung frei geblieben waren. Weiterhin gingen alle diejenigen Thiere, bei denen nach der Infection der Schlauch nicht angelegt wurde, bei denen jedoch nach 3-4. Stunden die Wunde energisch ausgebrannt wurde, ohne Ausnahme an Milzbrand zu Grunde.

Es geht aus diesen Versuchen hervor, dass die sofortige Umschnürung eines Gliedes einen vollkommenen Stillstand der auf die Wunde gebrachten Milzbrandbacillen zustande bringt, so dass man durch Ausbrennen der Wunde allein eine Vernichtung sämmtlicher Milzbrandbacillen bewirkt und damit den Ausbruch jeglicher Krankheitserscheinungen vereitelt.

Was für das Verhalten der Milzbrandbacillen in einer umschnürten Extremität gilt, wird sich mit Sicherheit auch auf alle übrigen in frische Wunden aufgenommenen Bacterien übertragen lassen. . Gerade der Milzbrand ist gewissermassen als Paradigma für die Untersuchung dieser Frage sehr gut, weil sich hier der Beweis eines vollkommenen Stillstandes der Bacterienbewegung, wie wir schon betont haben, mit Leichtigkeit führen lässt. 